\title{
Téoros
}

Revue de recherche en tourisme

\section{L’identité scientifique du tourisme} Un mythe ou une réalité en construction?

\section{Boualem Kadri}

Volume 27, numéro 1, printemps 2008

Science du tourisme ou études touristiques?

URI : https://id.erudit.org/iderudit/1070897ar

DOI : https://doi.org/10.7202/1070897ar

Aller au sommaire du numéro

Éditeur(s)

Université du Québec à Montréal

ISSN

0712-8657 (imprimé)

1923-2705 (numérique)

Découvrir la revue

Citer cet article

Kadri, B. (2008). L'identité scientifique du tourisme : un mythe ou une réalité en construction? Téoros, 27(1), 51-58. https://doi.org/10.7202/1070897ar d'utilisation que vous pouvez consulter en ligne.

https://apropos.erudit.org/fr/usagers/politique-dutilisation/ 


\section{L'identité scientifique du tourisme Un mythe ou une réalité en construction?}

\section{Boualem Kadri}

Célébré aux XVIII et $\mathrm{XIXe}$ siècles comme un art de distinction sociale par les élites aristocratique et bourgeoise, reconnu au $X X^{e}$ siècle et au début du XXIe en tant que pratique culturelle et outil économique d'importance, le tourisme peine toutefois à se définir une identité scientifique. Malgré un effort d'affirmation sur les plans universitaire (développement de programmes de maitrise et doctorat), associatif (existence d'organisations et associations internationales) et médiatique (diffusion des connaissances à travers des revues, des journaux et des rencontres scientifiques), nous relevons toutefois une fragmentation et une dispersion dans le processus de recherche en tourisme (diversité de disciplines et d'acteurs), mettant ainsi en évidence une absence de consen- sus sur le statut scientifique du tourisme. Qu'est-ce qui peut être à l'origine de ce manque de consensus ? Y a-t-il dans le développement de la pensée du tourisme une volonté de faire émerger une science et une discipline autonomes ? Quelle épistémologie envisager pour le tourisme? Le présent article vise à observer les différents éléments qui aideraient à cerner l'identité scientifique du tourisme.

\section{Le tourisme: une identité en question}

Le tourisme exprime tout d'abord une image globale marquée par une identité troublée sur plusieurs aspects.

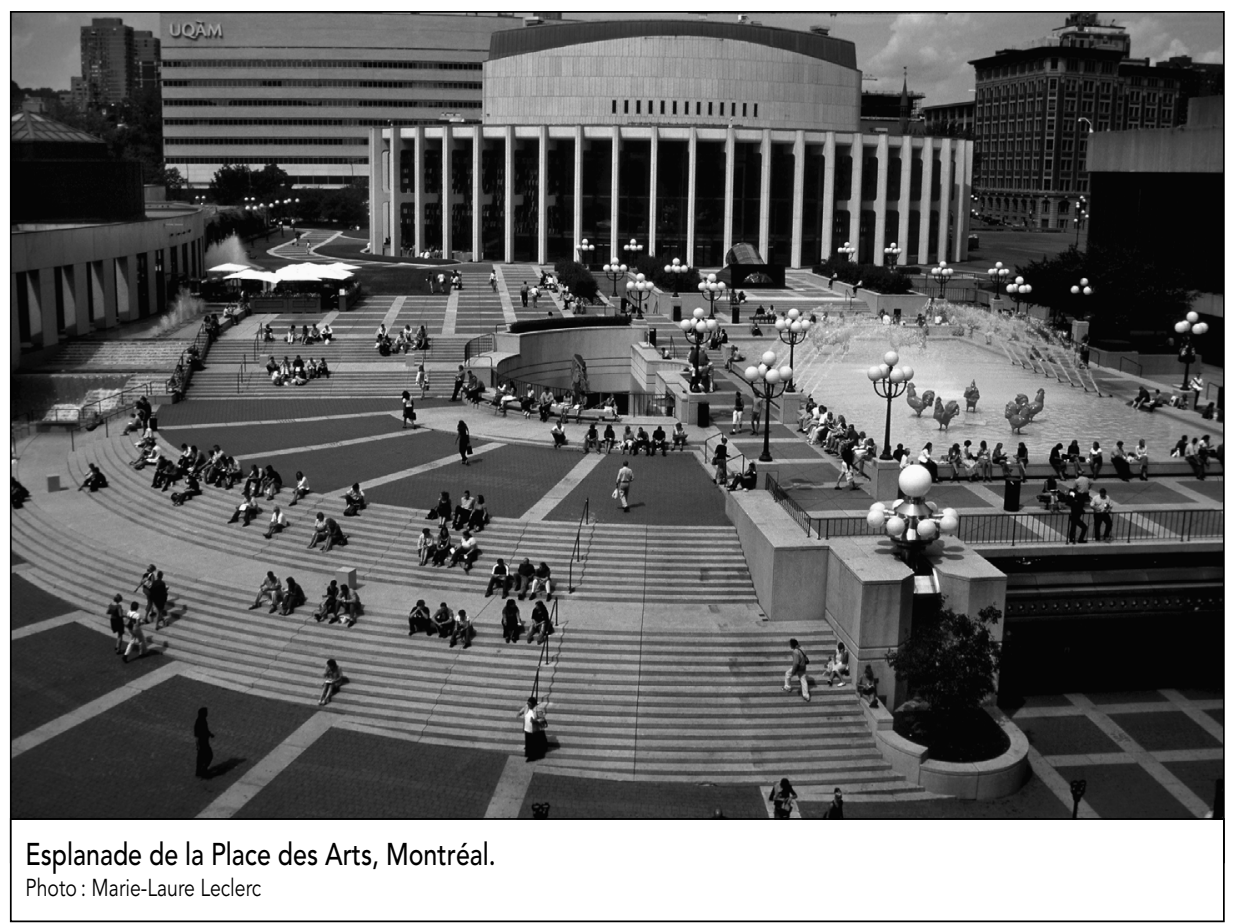

\section{La perception négative du tourisme, du touriste et du spécialiste}

Le tourisme, le touriste et le spécialiste qui pense le phénomène traînent une image réductrice et négative. Déjà, sur le plan sémantique, le mot "touriste» dans le Dictionnaire Larousse (2000) sert à identifier, bien sûr, celui qui voyage pour son plaisir, mais il est utilisé aussi pour qualifier les voyageurs qui choisissent la classe la plus économique (classe touriste) dans un mode de transport donné. Cette image catégorise déjà le touriste et la «masse » touristique, face au «voyageur » mythique. Cette problématique du mépris du touriste a été particulièrement bien mise en évidence par l'étude de Jean-Didier Urbain (1991) à travers les écrits antitouristiques des $X I X^{e}$ et $X X^{e}$ siècles, et rappelée par l'équipe MIT (Mobilité, Itinéraire, Tourismes) de Paris, qui s'interroge sur ce discours antitouristique: "Pourquoi tant de haine? » (2002). En effet, de nos jours encore, cette critique antitouristique se poursuit d'une manière assez virulente, par le biais d'écrits très médiatisés tels ceux de l'urbaniste Thierry Paquot (2001) et de l'historien Gérald Messadié (2003). Leur point commun est, d'une part, le mépris affiché envers le touriste consommateur effréné et, d'autre part, la perspective de voir le tourisme s'arrêter dans son développement.

Cette perception réductrice du tourisme et du touriste touche aussi celui qui se consacre à son étude. Si le mot touriste n'est pas utilisé que pour qualifier le spécialiste en «iste » s'intéressant au tourisme, bien heureusement, il sert néanmoins à le percevoir négativement par ceux souvent qui partagent la même origine disciplinaire. Par exemple, "les sociologues et les économistes (et les 
autres 'istes') spécialistes de la téorologie sont toujours perçus comme des 'touristes' à l'intérieur de leur propre discipline» (Stafford, 1992 : 44-46). L'Association française des experts et scientifiques du tourisme (AFEST, 2004) fait aussi remarquer la difficulté de la reconnaissance du travail scientifique en tourisme qui ne trouve sa marque que dans la discipline d'origine du chercheur.

La fragilité identitaire du tourisme s'exprime fortement dans cette perception négative, mais aussi par l'absence d'une définition reconnue de ce qu'est le phénomène touristique.

\section{La difficile émergence d'une définition reconnue et globale}

L'absence d'une définition reconnue par l'ensemble de la communauté de chercheurs montre cette difficulté d'obtenir un consensus et représente un autre handicap majeur dans la formation d'une identité scientifique du tourisme. II existe, depuis plus de quatre décennies, une variété de propositions où chaque discipline intéressée par l'étude du tourisme (anthropologie et psychologie entre autres) élabore une définition propre, mais réductrice aux aspects étudiés, produisant un certain « déficit sémiologique » (Boyer, 1999 : 22).

La complexité du phénomène, exprimée notamment par la diversité des intervenants (industrie, services, populations, touristes) et de leurs interrelations, influe sur la difficulté à fournir une définition globale acceptée par tous (Goeldner et Ritchie, 2006: 4-7). Toutefois, des tentatives de fournir des définitions regroupant l'ensemble des aspects du phénomène sont produites par certaines disciplines. En géographie, par exemple, on propose une perspective systémique rassemblant des aspects d'études dispersés dans les disciplines: "Le tourisme n'est ni une activité ou une pratique, un acteur ou un espace ou une institution: c'est l'ensemble mis en système. Et ce système comprend : touristes, lieux, territoires et réseaux touristiques, marché, pratiques, lois, valeurs et jeu des autres institutions sociales » (Knafou et Stock, 2003). Les spécialistes de la gestion, tels Charles $\mathrm{R}$. Goeldner et J.R. Brent Ritchie (2006 : 4-7), estiment aussi qu'il est nécessaire de prendre en considération plusieurs éléments pour construire une définition globale :
Thus tourism may be defined as the processes, activities, and outcomes arising from the relationships and the interactions among tourists, tourism suppliers, host government, host communities, and surrounding environments that are involved in the attracting and hosting of visitors.

Malgré cet effort d'intégration et de synthèse, ces définitions à l'aspect holistique sont perçues comme devant toujours être améliorées. C'est ce qui s'est passé, par exemple, dans le cas de la définition du tourisme de 1995 de RobertW. Mclntosh et Charles R. Goeldner, que John Tribe (1997) suggère de compléter par la prise en compte notamment des "environments ", ce qu'ils semblent avoir fait dans leur réédition de 2006 (Goeldner et Ritchie 2006: 4-7). L'apport améliorant et enrichissant la définition du tourisme apporte-t-il pour autant un consensus autour d'une définition acceptée par la communauté de chercheurs? Chaque définition est à améliorer, certes, mais elle continue néanmoins à porter la marque de fabrique d'une discipline. Toutefois, ce qui est partagé depuis quelques années, c'est la perception d'une complexité du phénomène et la nécessité de prendre en compte dans une définition globale ses diverses dimensions.

Il apparait paradoxal que le tourisme souffre de l'absence d'une définition "académique " reconnue par la communauté des chercheurs, alors que ces derniers utilisent en les critiquant les définitions d'organismes officiels qu'ils estiment répondre à un besoin institutionnel plus que scientifique. Les chercheurs en tourisme sont alors perçus comme prisonniers d'une certaine "confusion" liée aux visions institutionnelles (catégories statistiques) et entretenue par un système (pratiques, institutions et chercheurs) dans lequel le chercheur endosse une certaine responsabilité (Knafou, 2007: 9-20; Duhamel, 2007 : 351-358). Plusieurs spécialistes mettent ainsi en évidence la diversité des définitions et de la terminologie issues notamment d'organismes officiels (internationaux et nationaux) qui renforcent le manque de consensus scientifique (Goeldner et Ritchie 2006 : 4-7 ; Sacareau et Stock, 2003).

La difficulté à établir une définition commune est aussi liée à la diversité des disciplines agissant dans l'étude du tourisme. À titre d'exemple, Jafar Jafari (2001: 20-41) dénombre 21 départements ou disciplines uni- versitaires intervenant dans le champ du tourisme. À l'Université du Québec à Montréal, nous avons recensé 15 disciplines associées à la production des connaissances en tourisme (Kadri et Bédard, 2006 : 78-80). Cette situation exprime un autre paradoxe. D'une part, le tourisme est perçu comme futile et destructeur, mais il attire néanmoins l'intérêt de diverses disciplines. D'autre part, cette richesse disciplinaire produit aussi une difficulté à faire émerger une cohérence en termes de définition, de paradigmes et de méthodes d'approche.

Malgré ces difficultés, nous observons que la pensée en tourisme s'est développée d'une manière sensible depuis les années 1960.

\section{Le développement scientifique du tourisme : connaissances et reconnaissance}

La production de connaissances connaît une évolution importante depuis les années 1950-1960. J. Jafari (2001) a analysé l'évolution de la pensée en tourisme en décrivant quatre étapes et en mettant l'accent sur le fait que la production scientifique est surtout réalisée à partir des années 1990, notamment dans le milieu universitaire. Rappelons ces quatre étapes :

- Advocacy Platform - The Good (1960) : Le tourisme est perçu comme bénéfique pour la société et l'économie; c'est une analyse en termes d'impacts du tourisme, réalisée par des institutions et des firmes.

- Cautionary Platform - The Bad (1970) : Le tourisme est présenté comme un danger (pour la nature, la culture, l'économie). L'analyse est aussi effectuée en termes d'impacts.

- Adaptancy Platform - The How (1980) : C'est une vision centrée sur les ressources locales et la préservation, stimulant une analyse du tourisme en termes de développement de formes de tourisme davantage adaptées à la société et à l'individu,

- Knowlewdge-based-Platform - The Why (1990) : Contrairement aux étapes précédentes, celle-ci met en évidence le développement d'une vision globale du tourisme qui privilégie un contexte multidisciplinaire et vise "the formation of a scientific body of knowledge on tourism » (Jafari, 2001: 20-41). 
J. Jafari montre que ces modes de pensée coexistent encore et que le mode de compréhension du tourisme comme globalité n'émerge qu'à partir des années 1990. Toutefois, nous proposons de considérer que l'analyse du tourisme global avait commencé avant les années 1990. Par ailleurs, nous suggérons une approche de l'évolution de la pensée, non selon une approche segmentaire du tourisme (le bon, le mauvais...), mais dans une perspective de « reconnaissance " du phénomène comme étant important, en prenant en compte l'expérience touristique comme résultat d'un système.

Nous pouvons alors identifier trois stades d'analyse et de reconnaissance du tourisme dans son approche scientifique :

- Le stade de la reconnaissance idéologique du tourisme (1950-1970) : II s'agit ici d'une phase caractérisée par les jugements et les critiques envers le tourisme et le touriste. Par exemple, l'approche de l'expérience du touriste se fait sur fond de critique idéologique de la société bourgeoise et du modèle économique capitaliste. Divers auteurs ont développé cette vision idéologique, tels Roland Barthes (1957) et Daniel Boorstin (1963), ou Hans Magnus Enzensberger (1965), qui énonçait déjà en 1958 une « théorie du tourisme ». Cette vision idéologique du tourisme est également bien analysée par Olivier Burgelin (1967), qui s'appuie notamment sur les deux derniers auteurs. On approche déjà le tourisme comme un phénomène important, un système de représentation complexe (les mythes) qui nécessite de s'intéresser au caché, au symbolique (Barthes, 1957, Laurent 1973).

- Le stade de la reconnaissance symbolique du tourisme (fin 1970-1990) : C'est une période caractérisée par la compréhension du tourisme et du touriste. Par exemple, l'analyse anthropologique et sémiologique (Urbain, 1991) amorce une réconciliation entre le touriste et le voyageur : le regard du touriste est un « regard multiple », par opposition au regard prétentieux du voyageur. Rachid Amirou (1995) montre que le touriste se rapprocherait du pèlerin par le rituel de la situation de voyage et l'effort à accomplir dans la quête de sens. Dans les écrits nord-américains, l'approche du tourisme et du touriste, en ce qui concerne l'expérience touristique par exemple, met particulièrement l'accent sur la globalité de l'expérience, perçue comme une "production culturelle» de la société (MacCannel, 1976), une totalité complexe «espace-temps-culture » (Jafari, 1988), alors que l'industrie touristique réduit cette complexité à une banalisation par l'offre où tout est inclus (Laplante, 1999). Or, l'approche du tourisme selon une vision de globalité et de complexité est essentielle à la formation des connaissances scientifiques.

- Le stade de la reconnaissance de la complexité du phénomène (fin 19902000 et plus) : C'est la reconnaissance ici que le phénomène touristique ne peut être réduit à quelques aspects analysés par les sciences sociales selon leurs approches (le touriste et sa motivation, le tourisme et l'offre d'équipements et de services, le tourisme en termes de flux, etc.). Cette vision de la complexité se renforce notamment par l'apport d'autres sciences ouvertes, comme le tourisme, aux changements et aux transformations (dont l'écologie). C'est alors nécessaire de comprendre le tourisme comme un tout, comme un système en interaction. On relèvera, par exemple, l'approche géographique qui définit le tourisme comme un système à plusieurs variables (Knafou et al., 1997 ; Knafou et Stock, 2003) ; l'intérêt de faire appel aux théories de la complexité et du chaos pour comprendre le fonctionnement du tourisme défini comme un système chaotique (McKercher, 1999) ou pour proposer un paradigme fédérateur (Dewailly 2006); l'importance d'intégrer les analyses engagées par des sciences tel l'environnement qui, comme le tourisme, fait de la "durabilité»sa perspective de développement (Farrel et Twining-Ward, 2003 : 274-295).

Cette évolution de la production des connaissances s'appuie sur des intervenants importants, dont l'université et la revue de recherche. En effet, on compte aujourd'hui de nombreux programmes dans le monde. En France, par exemple, ils sont tellement nombreux qu'il devient nécessaire de créer un programme fédérateur pour faciliter leur identification (Baron-Yelles, 2001). Dans plusieurs universités du monde anglo-saxon (ÉtatsUnis, Angleterre, Australie) il existe aussi des programmes de deuxième et de troisième cycles en tourisme. En Australie, notamment, on observe que 25 universités offrent des cours de tourisme (Leiper, 2000: 805-809).
Au Québec, deux programmes de premier et de deuxième cycles de type professionnel ont vu le jour depuis 1976, mais la production scientifique (maîtrise et doctorat) est réalisée par d'autres disciplines, montrant ainsi leur domination sur celle du tourisme (Kadri et Bédard, 2006: 78-80). Le développement d'un programme de maîtrise avec profil de recherche et d'un programme doctoral en tourisme, utilisant les nouvelles technologies, sont prévus pour les prochaines années.

La construction des connaissances en tourisme est assurée aussi par la revue scientifique qui joue un rôle essentiel dans le processus de diffusion de connaissances, mais aussi de reconnaissance du tourisme. II existe un nombre important de revues spécialisées. Ainsi, J. Jafari (2001) dénombre 40 journaux et revues scientifiques en langue anglaise, dont: Annals of Tourism Research et Journal of Travel Research. La première, de la University of Wisconsin-Stout (USA), qui existe depuis 1973, a deux orientations importantes: les constructions théoriques et méthodologiques et les aspects du développement et des impacts (Honggen et Stephen, 2006 : 490-507).

Du côté des revues de langue française, citons notamment: Espaces (France), Téoros et Loisirs et Société (Québec). Téoros, revue de recherche fondée en 1982 à l'UQÀM, a aussi deux orientations principales: d'une part, capter et mobiliser l'attention de l'industrie touristique aux problèmes du tourisme; d'autre part, saisir et diffuser l'image d'un tourisme au Québec, tant sur le plan interne (renforcement de l'identité culturelle) que sur le plan externe (analyse de produits originaux pour des clientèles diversifiées) (Bédard et Kadri, 2000: 67-76). La revue Loisirs et Société de I'Université du Québec à TroisRivières, fondée en 1978, consacrée au développement scientifique du loisir, apporte un complément dans la reconnaissance scientifique s'intéressant à la dimension épistémologique du tourisme, orientation investie par Téoros dans les années 1980-1990 et peu à peu retrouvée aujourd'hui.

La revue joue un rôle fondamental dans la construction de l'identité scientifique du tourisme et émerge comme un espace «transitionnel » de rencontre d'acteurs et de disciplines divers où peuvent se façonner des collaborations disciplinaires nouvelles débouchant sur une épistémologie particulière. 
C'est donc le statut scientifique du tourisme qui est questionné ainsi que la dimension épistémologique de ce champ de recherche.

\section{Quelle épistémologie pour le tourisme?}

\section{La difficulté d'établir un statut - discipline, science ou champ d'études?}

Les controverses sur le statut du tourisme dans la communauté des chercheurs alimentent davantage cette quête d'identité scientifique et démontrent un manque de consensus sur la question. Les positions concernant la perception du tourisme comme discipline et science restent très partagées. Des propositions de constitution d'une nouvelle science, celle du tourisme, nommée et identifiée comme telle, ont été avancées depuis les années 1980. Ainsi, en 1981, Neil Leiper propose d'appeler cette nouvelle discipline tourology, pour soutenir la formation et l'enseignement pluridisciplinaires en tourisme et adossée à une théorie générale (rapporté par Tribe, 1997, qui montre que le terme n'a pas été utilisé depuis par la communauté). Au Québec, Jean Stafford (1985; 1992) propose la téorologie, du grec teoros qui veut dire le "voyage ", comme étant le champ d'étude et de construction des connaissances scientifiques du tourisme: "La téorologie se définira par cette capacité d'intégrer des éléments disparates liés à l'espace et au temps, à l'économie et à la politique, à la psychologie et à la gestion et enfin à la culture et au patrimoine. ” (Stafford, 1992: 44-46) Ce champ de connaissances du tourisme est identifié comme un espace d'intégration d'approches multidimensionnelles des niveaux de la réalité complexe que représente le tourisme. De ce point de vue, il s'agit plus d'identifier un champ d'études que de créer une science.

Un autre terme fait aussi son apparition: face à la complexité du phénomène, certains voient la nécessité d'établir une discipline autonome, la tourismologie, qui étudierait le tourisme dans sa globalité et non d'une façon fragmentée comme le font les diverses disciplines (Jovicic, 1988, rapporté par Echtner et Tazim, 1997 : 868-883). Cette appellation d'une science du tourisme se retrouvera près de vingt années plus tard en France, sans priorité épistémologique, mais avec une perspective scientifique limitée à l'industrie et aux métiers du tourisme (Hoerner, 2000).
Cette proposition de constitution d'une science du tourisme a stimulé une polémique entre chercheurs, souvent d'une même origine disciplinaire (la géographie). Ce débat a fait observer les rapports tendus entre les tenants des disciplines constituées perçus comme conformistes et les experts liés à des associations (Association Mondiale pour la Formation Hôtelière et Touristique / AMFORHT, Association française des experts et scientifiques du tourisme / AFEST) qui apportent leur soutien à la proposition d'une constitution de la tourismologie. D'autres, tels Georges Cazes (2001) et Rémy Knafou (2005), avouent que la reconnaissance scientifique est en train de se construire et ils estiment alors qu'il est prématuré de proclamer la naissance d'une science. Si les études touristiques évoluent vers la constitution d'une discipline distincte, elles ont besoin surtout de développer rapidement une interdisciplinarité (Echtner et Jamal, 1997 : 868-883).

De l'avis de certains tenants des sciences de la gestion, la reconnaissance d'un statut disciplinaire du tourisme est affiché. Selon C.R. Goeldner et J.R.B. Ritchie (2006), "Modern tourism is a discipline". Ils mettent par ailleurs en évidence la difficulté de cette discipline de se constituer comme telle, du fait de l'intérêt récent qu'on lui porte et de l'absence d'une définition commune. Cette motivation à reconnaître le tourisme comme discipline serait peut-être à rattacher au statut des sciences de la gestion qui construisent aussi leur propre identité scientifique (Martinet,1990; David et al., 2000 ; Le Moigne, 2001).

Face à ces propositions dites favorables à la reconnaissance d'un certain statut scientifique, d'autres chercheurs s'opposent farouchement. Ainsi, on relèvera la perspective sans nuance de René Baretje (2007) à l'idée d'une science du tourisme: "Le tourisme n'est et ne sera jamais une science mais une activité. » J. Tribe (1997), auteur de quelques essais épistémologiques sur le sujet, exercice important et au demeurant rare, refuse les statuts de discipline et de science et avance l'idée d' « indiscipline » du tourisme. II perçoit le tourisme comme un objet d'études (un champ, donc moins de cohérence) plutôt qu'une discipline d'études, le reléguant par le fait même à un état ou à une identité scientifique de moindre envergure: "Tourism studies seem likely to remain in a pre-paradigmatic phase [...] but this should not be seen as a problem. Rather tou- rism studies should recognize and celebrate its diversity. » (Tribe, 1997) Ce débat sur le statut du tourisme comme discipline a aussi déclenché, en 2000, une polémique entre deux auteurs anglo-saxons, Neil Leiper et John Tribe (voir références en bibliographie).

Pour rejeter cette reconnaissance en termes de statut scientifique, Tribe (1997) fait référence à certains critères de type positiviste utilisés dans l'évaluation de la science: emprunts conceptuels à d'autres disciplines, absence de cadre théorique cohérent, problèmes du tourisme non résolus par les outils propres au tourisme.

Bref, le tourisme ne possède rien en propre. Avec des conditions épistémologiques issues du « réalisme critique ", le tourisme ne résiste pas et se trouve alors ignoré ou même exclu de «l'arbre de la connaissance " (en référence à Auguste Comte) ${ }^{1}$ comme l'ont été auparavant d'autres disciplines (telles l'économie politique, la psychologie) (Le Moigne, 2001: 173). Le Moigne, qui s'est notamment intéressé à l'épistémologie des sciences de la gestion, dont certaines ressemblances avec le tourisme seront relevées plus loin, montre que l'on s'appuie sur des principes poppériens (comme le principe de réfutabilité) pour exclure de "l'arbre de la connaissance» d'autres disciplines. Le tourisme se retrouve donc bien plus dans une position de champ d'études que beaucoup de spécialistes partagent (ex: Jafari 1988, 2001 ; Tribe, 1997 ; Cazes, 2001) et accessible à toute incursion extérieure, que dans celui d'une possible discipline ou science. Que faire face aux critères de scientificité positivistes?

Le travail épistémologique effectué dans les sciences de la gestion par Jean-Louis Le Moigne (2001), Alain Charles Martinet (1990), Albert David, Armand Hatchuel et Romain Laufer (2000) peut-il nous éclairer et orienter l'effort à consentir en tourisme?

\section{La perspective d'une épistémologie interne et constructiviste dans les sciences sociales - L'exemple des sciences de la gestion}

L'exemple de la construction épistémologique dans les sciences de gestion peut-il éclairer celle en tourisme? Revisitant les travaux du philosophe Gaston Bacherlard, notamment pour son ouvrage Le nouvel esprit scientifique (1934), J.-L. Le Moigne 
(2001) invite à poursuivre l'effort de refondation épistémologique (non cartésienne) développée par G. Bachelard et poursuivie par Jean Piaget (1967). II s'agit ici de la nécessité de revoir la pertinence épistémologique d'évaluation d'une science, d'une discipline. Un « grand courage épistémologique " est encore requis des sciences sociales et humaines pour revoir les critères d'évaluation concernant leurs pratiques scientifiques et déterminer leur légitimité épistémologique (Le Moigne, 2001).

J.-L. Le Moigne montre que les sciences de la gestion ont tenté d'assurer leur légitimité épistémologique par des stratégies diverses dans les milieux universitaires : présentation des sciences de la gestion comme une discipline carrefour d'autres disciplines, développement de corporations liées à d'autres (école de commerce, d'ingénieurs), création de réseaux (nationaux et internationaux) en matière de diffusion de connaissances (conférences, revues), développement de programmes de formation supérieure manifestant l'existence d'une discipline en mouvement. Cette discipline importe des concepts d'autres disciplines et ne présente pas de théorie propre pouvant satisfaire «les canons de la validation positiviste » et les principes de l'hypothético-déduction, y compris la fameuse théorie de la contingence organisationnelle de P.R. Lawrence et J.W. Lorsh, dont la validité ne fut réalisée que sur six entreprises américaines (Le Moigne, 2001 : 223). Cette «incongruité épistémologique ", selon Le Moigne, est liée au fait que la validation de la théorie est légitimée sur une réputation externe: celle du chercheur d'une autre discipline ou celle de la méthode produite ailleurs. Ne répondant pas aux critères de scientificité positiviste, les sciences de gestion sont-elles menacées dans leur avenir, scientifique ou autre?

Selon J.L. Le Moigne (2001 : 237), pour « incongrue que soit en apparence sa prétention à un statut épistémologique correctement facturé de discipline scientifique, la science de gestion manifeste la plupart des comportements habituels d'une science normale $^{2}$ » (programmes de formation et de recherche, écoles de pensée, reconnaissance internationale de certains chercheurs tel Herbert A. Simon (prix Nobel, 1978), revues, colloques, fédérations et sociétés savantes). Le processus interne de légitimité épistémologique a une importance considérable pour les sciences de la gestion:
«L'incongruité épistémologique » devient alors un instrument original de diagnostic de scientificité [...] Si l'épistémologie sur laquelle se fondent apparemment les sciences de gestion apparaît incongrue (ce qui n'est pas convenable, ou qui ne convient pas à la situation scientifique de la discipline) et que nonobstant, la discipline manifeste durablement une vitalité suffisante pour que les communautés scientifiques ne la bannissent pas pour charlatanisme, on peut s'interroger sur l'origine de ce manque de confiance: est-ce la discipline qui n'est pas congrue à l'épistémologie de référence, ou est-ce la référence épistémologique qui n'est pas congrue à la discipline? Rigueur intellectuelle autant que pragmatisme nous incitent aujourd'hui à suivre l'exemple d'Yves Barel et à reconsidérer la pertinence de la référence épistémologique à l'aune de laquelle nos sociétés tentent d'évaluer la scientificité de la discipline (et plus généralement, le nombre de "nouvelles sciences"). (Le Moigne, 2001 : 239)

Les sciences de la gestion s'interrogent sur leurs méthodes et leurs pratiques et construisent ainsi de nouveaux discours et «gagnent à forger leur propre épistémologie adaptée à leur intention et aux 'objets' qu'elles étudient et conçoivent » (Martinet, 1990 : 9-29).

Depuis la fin des années 1960, la question épistémologique dans les sciences de la gestion est investie notamment aux États-Unis par un des plus célèbres théoriciens, H.A. Simon. En France, depuis notamment la fin des années 1980, une importante réflexion épistémologique est entreprise (Martinet, 1990 ; David et al., 2000 ; Le Moigne 2001). Poursuivant le débat épistémologique initié par Martinet (1990), A. David, A. Hatchuel et R. Laufer (2000), tentent de refonder les sciences de gestion. Ils observent certes la réussite et la reconnaissance des sciences de la gestion à travers leur pratique professionnelle et l'ancrage social de l'acteur « entreprise », mais s'interrogent sur leur " déficit d'identité », fondé notamment sur l'exigence d'établir un objet propre à la discipline. Par ailleurs, les auteurs observent, comme on le ferait pour le tourisme aussi, la fragmentation de la recherche qui entraîne un manque de cohésion théorique et de consensus dans la communauté des spécialistes. C'est alors «l'absence d'un champ fédérateur [qui] rend les sciences de gestion poreuses " (ex: absence de critères propres de scientificité pour vérifier les emprunts des autres, ce que reprochait J. Tribe [1997] au tourisme). David, Hatchuel et Laufer perçoivent pour les sciences de gestion « une identité scientifique nouvelle» rejetant la vision d'une discipline réduite à un ensemble d'outils spécialisés, la division entre les courants de pensée et les querelles méthodologiques internes et externes. Les sciences de gestion construisent leur champ de recherches unifié sur une refonte des problématiques. C'est alors " une science dont l'objet ne serait ni un type d'organisations, ni un type de phénomènes, ni un ensemble de faits, mais plutôt une classe de problématiques constitutives de toute action collective [...] Leur objet de recherche ne se réduit en définitive ni à une sociologie appliquée, ni à une économie appliquée» (David et al., 2000: 21-26). Les sciences de gestion tentent ainsi de s' « autonomiser » des autres sciences sociales, mais cette volonté de construire une identité scientifique aurait un prix, celui de voir "des mutations de la notion même de 'gestion' ", tout en procurant une perspective importante, celle d'éclairer "des questions transversales à l'ensemble des sciences sociales ". Ainsi, les sciences de gestion se libèrent des autres sciences pour mieux y retourner et les enrichir. Cette liberté vient de leur statut à être "entre... et entre... ». Martinet (1990 : 9-29) écrit à ce propos : "Les sciences de gestion doivent naviguer entre les écueils de la technique aveugle, de l'idéologie pure, de la sophistique généralisée et du scientisme mutilant. À ce prix, elles échappent à la remorque des 'vraies sciences'. Elles peuvent même contribuer à la renaissance des méthodes. ” Les sciences de la gestion sont conséquemment dans « une mutation essentielle tant pour elle-même que pour les autres sciences sociales ou pour la société 》 (David et al., $2000: 21-26)$.

Quel parallèle peut-on faire avec le tourisme? On pourra observer que le tourisme présente certaines caractéristiques similaires aux sciences de la gestion : présence de nombreux programmes de deuxième et de troisième cycles, de revues scientifiques, de colloques, de corporations scientifiques internationales. Si l'on enregistre aussi une fragmentation dans la recherche, une importation importante de concepts, on observera depuis deux décennies un effort considérable dans l'amorce d'une certaine épistémologie interne. 
Ainsi, J. Stafford, économiste et sociologue, s'est investi longuement dans une épistémologie du tourisme. II a présenté les nécessités épistémologiques pour asseoir une science du tourisme, en s'intéressant à la critique des paradigmes de la recherche (hypothèses, concept, méthodologie). II identifie quatre grands paradigmes qui structurent la recherche en tourisme: 1) le paradigme nominaliste (centré sur la cueillette des données, d'un niveau descriptif) ; 2) le paradigme économico-spatial (analyse de la demande, avec un caractère explicatif); 3) le paradigme culturaliste (étude des rapports sociaux complexes à partir des méthodes de la sociologie, de la sémiologie, de l'anthropologie); et 4) le paradigme normatif (orienté vers ce que doit être le tourisme, avec un aspect idéologique) (Stafford, 1985 : 459-560). Seuls les paradigmes culturalistes et économicospatiaux apparaissent comme ayant un pro-

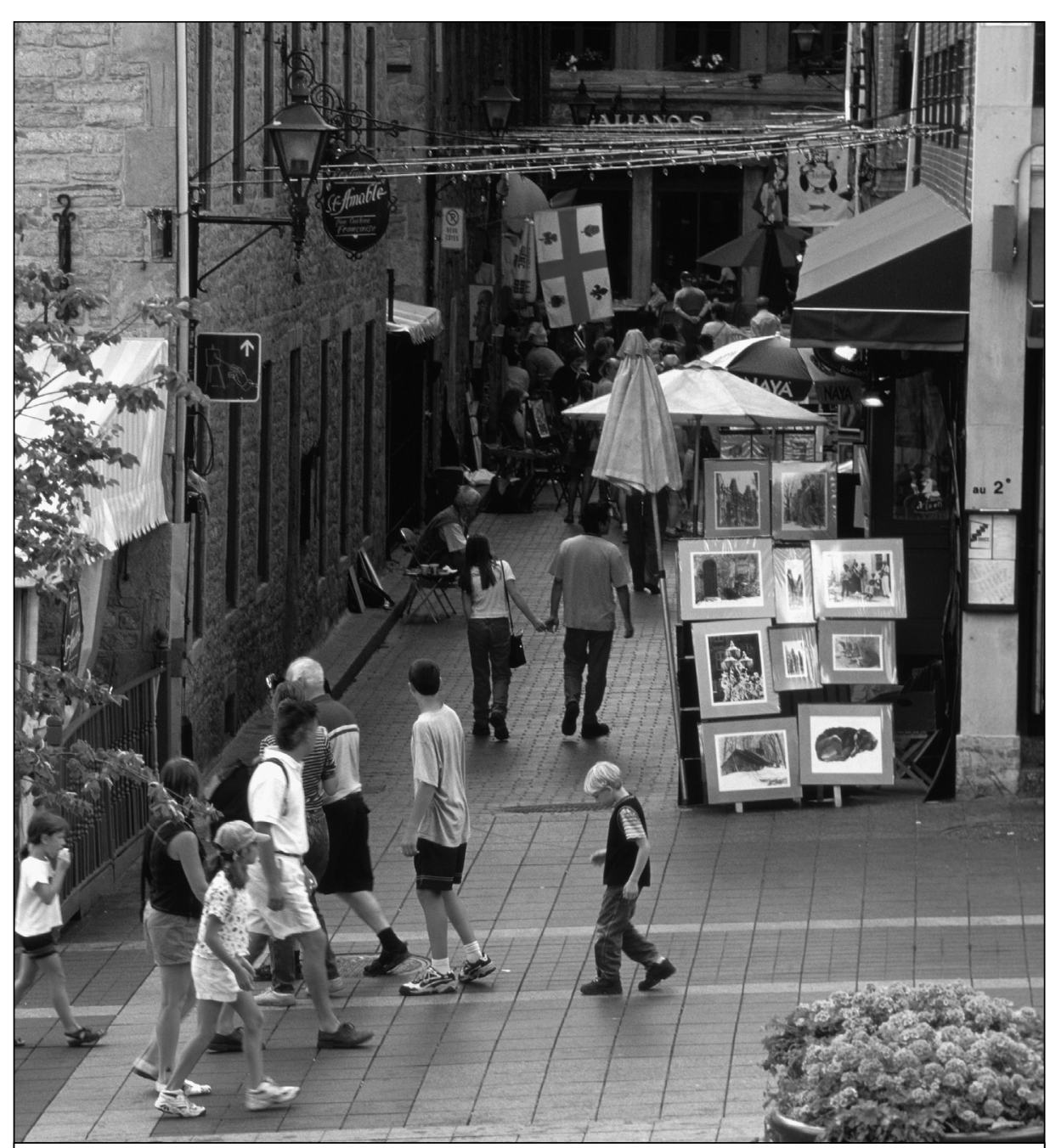

Promenade dans le Vieux-Montréal, Place Jacques-Cartier, Montréal.

Photo: Tourisme-Québec

cessus scientifique. Ce travail scientifique suscitera aussi des interrogations quant à la nécessité ou la justification d'un investissement épistémologique en tourisme (Bodson et Stafford, 1988: 3-5), compte tenu du manque de cohésion théorique observé et de la forte influence des sciences appliquées (satisfaction, contrôle). L'auteur estime qu'il est plus approprié aujourd'hui de déterminer ce qu'est le champ du tourisme et surtout de définir au sein de la communauté scientifique les problématiques du tourisme qui doivent être étudiées (Stafford, 2003 : 87-95). Un certain courage épistémologique est demandé aux chercheurs qui seraient «prêts à jouer le jeu des problématiques et des solutions » (ibid.). Déjà, Thomas Samuel Kuhn (1983 : 48) montrait que le travail de la science signifie d'abord de concentrer "son attention sur un secteur limité de problèmes ».
C'est l'effort épistémologique de refonte déjà observé dans les sciences de gestion, mais aussi le " courage épistémologique ", qui seraient entre autres marquants dans le développement d'un champ de recherche. L'intérêt d'une perspective fédératrice chez les chercheurs en tourisme, comme en sciences de gestion est alors recherché.

Jean-Michel Dewailly (2006), dans un essai qui se rapproche de la démarche de l'épistémologie interne, propose la perspective d'un paradigme fédérateur qui prendrait en compte notamment les dimensions anthropologique et géographique en élargissant l'horizon historique du tourisme (à d'autres périodes que celle industrielle en Occident). C'est l'idée de "pérégrinité » avec sa dimension d'universalité. Le paradigme proposé à la communauté scientifique a une portée « fédératrice» :

Le paradigme fédérateur de ces chercheurs pourrait être l'étude des relations entre l'être humain en situation de pérégrinité et les espaces et les sociétés. Une formulation aussi large inclut tous les temps, tous les lieux, toutes les échelles, toutes les cultures, toutes les disciplines, et a donc vocation à être explorée de façon scientifique universelle. (Dewailly, 2006: 198)

Ici, l'auteur rejoint l'archéologue Érik Langevin (2000: 9-13) qui montre le déplacement d'individus dans la période préhistorique, dans le contexte des Amérindiens au Québec, où « le tourisme de la préhistoire » avait pour but le ressourcement à la fois physique, psychique et cognitif à travers la rencontre, lors de cycle de transhumance, avec d'autres groupes sociaux qui avaient d'autres habitudes culturelles.

Le phénomène touristique exprime ainsi une situation de complexité, dont l'étude nécessite des changements conceptuels et méthodologiques. H. Brian Farrel et Louise Twining-Ward (2003) montrent qu'il existe un problème fondamental dans l'étude du fonctionnement du système du tourisme: c'est un fonctionnement non linéaire qui est analysé selon des approches traditionnelles non adaptées de type déterministe. De ce point de vue, la perspective de recherche devra faire un saut qualitatif et opérer une véritable transition conceptuelle où les disciplines intervenantes devront plus collaborer que dominer, dans un contexte constitué d'incertitude et de complexité : 
To move tourism towards such a transition, researchers, consultants, managers, and stakeholders need to understand complex systems through integrative and nonlinear approaches; otherwise progresses will be hampered and results distorted, incomplete and devoid of full meaning. Transdisciplinarity is desirable and interdisciplinarity is essential, with building desperately needed bridges as a goal. (Idem : 1275-1295)

L'exemple des sciences de gestion et d'autres sciences confrontées à la complexité (sciences de l'environnement, notamment) éclaire le processus de refonte épistémologique et les démarches à entreprendre en tourisme afin d'atteindre l'autonomie scientifique.

Le tourisme n'est pas seulement un outil au service d'une économie; son étude peut aussi enrichir d'autres disciplines, la géographie par exemple, comme le montrent Knafou et al. (1997:193-213): "L'analyse du tourisme est donc susceptible d'éclairer, d'une manière nouvelle, les outils et concepts de la géographie, et par la même occasion de produire de nouveaux outils pour saisir la mutation en cours ", celle des rapports du touriste aux lieux touristiques. Bien plus, le travail des chercheurs en tourisme peut avoir une contribution fondamentale, celle de "lire le monde' à l'aide de leur vision particulière dont chacun pourra reconnaître la spécificité et la pertinence. » (Cazes, 2001 :16-19)

La nouvelle épistémologie qui se construit de l'intérieur des sciences sociales permet la construction de leur identité scientifique. Comme le montre J. Piaget, cette démarche épistémologique ne se réduit pas à une simple réflexion sur la connaissance, elle est la conséquence de crises dans le processus de construction des connaissances nécessitant de s'interroger sur la valeur épistémologique des outils conceptuels utilisés :

Dans de tels cas, la critique épistémologique cesse de constituer une simple réflexion sur la science : elle devient alors un instrument d'un progrès scientifique en tant qu'organisation intérieure des fondements, et surtout en tant qu'élaborée par ceux-là mêmes qui utiliseront ces fondements et qui savent donc de quoi ils ont besoin au lieu de les recevoir du dehors à titre de présents généreux, mais peu utilisables et parfois encombrants» (Piaget, $1967: 51)$.
À ce sujet, Gilles Pronovost (1999: 1-13) a montré que le champ du tourisme prenait des risques lorsque des transferts de concepts souvent dépassés des sciences du loisir étaient effectués vers le tourisme.

Nous avons vu plus haut comment les sciences de gestion se construisent sur cette approche de l'épistémologie interne et la nécessité pour le tourisme d'en faire de même, processus déjà commencé, si nous nous fions aux réflexions de certains auteurs (Stafford, 1985 ; 1992 ; 2003 ; Tribe 1997 ; Farrel et Twining-Ward, 2003 ; Knafou, 2007 ; Dewailly, 2006).

Cette exigence épistémologique n'est pas limitée à certaines sciences, elle concerne la science d'une façon générale et, notamment, ses rapports avec la société. Aujourd'hui, le contexte de complexité et d'incertitude influe sur le mode de production des connaissances et la relation entre la science et la société, au point où l'on avance l'idée de repenser les fondements épistémologiques de la connaissance (Gibbons et al. 1994 ; Nowotny et al., 2003).

\section{Conclusion}

Le fait nouveau observé depuis près de deux décennies est que le tourisme stimule un intérêt de plus en plus grand en ce qui concerne son identité scientifique (critique épistémologique, réflexion sur son apport aux autres sciences et la société). Ce résultat vient démentir en partie l'observation de Marc Boyer (1999; 13) qui relevait que « La plupart des ouvrages, qui depuis une trentaine d'années, ont dit faire l'étude du tourisme ne sont pas allés au-delà du rappel de l'évidence: le tourisme est une migration de personnes libres ».

Cet aboutissement positif dans le développement scientifique du tourisme se heurte néanmoins à une image réductrice et négative qui continue encore de dévaloriser le tourisme, le touriste et le spécialiste. Toutefois, cela ne doit pas limiter "le courage épistémologique ", qui consiste à poursuivre la construction d'une épistémologie interne au tourisme et à définir les problématiques importantes, à réaliser les collaborations entre diverses sciences dans une perspective de " projet de connaissance » et non « d'objet de connaissance " (Le Moigne, 2001). En ce sens, l'interdisciplinarité, la transdisciplinarité, permettrait au tourisme de réduire son handi- cap épistémologique vis-à-vis des disciplines traditionnelles, dans la mesure où ces dernières limiteraient leur exercice de domination par leur participation au « projet de connaissance ", permettant au tourisme d'intervenir comme un partenaire scientifique à part entière. La problématique de l'identité scientifique ne concerne pas seulement «les nouvelles sciences ». Les sciences sociales sont ellesmêmes dans un état de mutation sur le plan scientifique: « Une formidable mutation, au fil de laquelle les outils théoriques et les orientations d'il y a trente-cinq ou quarante ans se sont déstructurés et sont dans l'ensemble entrés en crise, tandis que des outils et des orientations nouvelles s'ébauchaient. ". (Wieviorka), 2007 : 9-21). Selon HervieuLéger (2007), l'interdisciplinarité reste un vœu pieux pour les sciences sociales, qui restent enfermées dans "une incertitude identitaire ", alors que les disciplines nouvelles telle l'écologie scientifique assurent un dialogue interdisciplinaire. Les nouvelles sciences seraientelles mieux disposées aux changements épistémologiques et méthodologiques ? Ces divers aspects ouvriraient des perspectives de reconnaissance d'autres sciences émergentes, telle celle du tourisme, qui auraient aussi un nouveau rôle celui d'enrichir les sciences anciennes par l'épistémologie constructiviste qu'elles élaborent. «Retournement paradoxal en apparence, moins audacieux qu'il n'y paraît, qui conduira à proposer aux nouvelles sciences de devenir les inspiratrices du renouveau des anciennes disciplines. " (Le Moigne, $1990: 81-140$ )

Boualem Kadri est professeur associé au Département d'études urbaines et touristiques de l'Université du Québec à Montréal.

\section{Notes}

1 Selon Le Moigne (2001) : en référence à Auguste Comte qui l'a conçu au XIX ${ }^{e}$ siècle. Cet auteur s'interroge sur la présence encore forte de la métaphore de l'arbre de la connaissance.

2 Souligné par l'auteur.

\section{Bibliographie}

AFEST (2004), « Pour un champ disciplinaire autonome du tourisme", Association française des experts et scientifiques du tourisme, [http://www.afest.org/breve40.html], consulté en janvier 2008.

Amirou, Rachid (1995), Imaginaire touristique et sociabilités du voyage, Paris, Presses universitaires de France. 
Bacherlard, Gaston (1934), Le nouvel esprit scientifique, Paris, Presses universitaires de France.

Baretje, René (2007), Centre international de recherches et d'études touristiques, [http:// www.ciret-tourism.com/the_ciret/banques_ donnees_ciret.html], consulté en décembre 2007.

Baron-Yelles, Nacima (2001), «Fédérer les formations universitaires en tourisme et loisirs ", Revue Espaces, no 187, p. 46-53.

Barthes, Roland (1957), Mythologies, Paris, Éditions du Seuil.

Bédard, François et Boualem Kadri (2000), " La recherche en tourisme en Amérique du Nord francophone et anglo-saxonne à travers quelques revues. État des lieux ", Actes du colloque de Foix, 2-3 mai, La recherche en tourisme, Paris, Éditions Jacques Lanore, p. 67-78.

Bodson, Paul et Jean Stafford (1988), "Le paradigme économique en tourisme", Téoros, vol. 17, n³, p. 3-5.

Boorstin, Daniel J. (1963), L'image ou ce qu'il advint du rêve américain, Paris, Julliard.

Boyer, Marc (1999), Le tourisme de l'an 2000, Lyon, Presses universitaires de Lyon.

Burgelin, Olivier (1967), "Le tourisme jugé ", Communications, Dossier Vacances et tourisme, vol. 10, n 10, p. 65-96.

Cazes, Georges (2001), "À propos de tourismologie. La science par autoproclamation ", Revue Espaces, nº 178, juillet-août, p. 16-19.

David, Albert, Armand Hatchuel et Romain Laufer (dir.) (2000), "Introduction", dans Les nouvelles fondations des sciences de gestion. Éléments d'épistémologie de la recherche en management, Paris, Vuibert et Fondation nationale pour l'enseignement de la gestion des entreprises.

Dewailly, Jean-Michel (2006), Géographie et tourisme. Pérégrinité ou chaos?, Paris, L'Harmattan.

Duhamel, Philippe (2007), «Alors, un énième livre sur le "tourisme urbain", dans Philippe Duhamel et Rémi Knafou (dir.), Les mondes urbains du tourisme, Paris, Éditions Belin, p. 351-358.

Echtner, M. Charlotte et Tazim B. Jamal (1997). "The Disciplinary Dilemma of Tourism Studies ». Annals of Tourism Research, vol. 24, $n^{\circ} 4$, p. 868-883.

Enzensberger, Hans Magnus (1965), « Une théorie du tourisme ", Culture ou mise en condition?, Paris, Lettres nouvelles.

Équipe MIT (Mobilité, Itinéraire, Tourismes) (2002), "Pourquoi tant de haine?", Tourismes 1 , Paris, Belin, p. 11-75.

Farrel, H. Bryan et Louise Twining-Ward (2003), "Reconceptualizing Tourism", Annals of Tourism Research, vol. 2, n 3, p. 274-275.
Gibbons, Michael (1994), The New Production of Knowledge - The Dynamics of Science and Research in Contemporary Societies, Sage, London.

Goeldner, Charles R., et J.R. Brent Ritchie (2006), Tourism: Principles, Practices, Philosophies, Hoboken (N.J.), John Wiley \& Sons.

Hervieu-Léger, Danièle (2007), "Sciences sociales et autres sciences : quelles interfaces ? ", dans Michel Wieviorka (dir.), Les sciences sociales en mutation, Paris, Éditions sciences humaines, p. 239-248.

Hoerner, Jean-Michel (2000), «Pour la reconnaissance d'une science touristique ", Revue Espaces, n 173, juillet-août, p. 18-20.

Honggen, Xiao et Stephen L.J. Smith (2006), "The Making of Tourism Research. Insights from a Social Sciences Journal ", Annals of Tourism Research, vol. 24, n 3, p. 490-607.

Jafari, Jafari (1988), « Le système du touriste : modèles socioculturels en vue d'applications pratiques et théoriques", Revue Loisir et Société, vol. 11, no 1, p. 59-79.

Jafari, Jafar (2001), "The Scientification of Tourism ", dans Valene L. Smith et Maryann Brent (dir.), Hosts and Guests Revisited: Tourism Issues of the $21^{\text {st }}$ Century, New York, Cognizant Communication Corporation, p. 2941.

Jovicic, Zivodin (1988), «A Plea for Tourismological Theory and Methodology", Revue de Tourisme, vol. 43, n 3, p. 2-5.

Kadri, Boualem et François Bédard (2006), « La construction des connaissances en tourisme à l'université : la production de mémoires et thèses liés au tourisme à l'UQAM (1978 à 2005) ", Téoros, vol. 25, n², p. 78-80.

Knafou, Rémy (2005), " La recherche en tourisme s'organise ", Revue Espaces, n²25, avril, p. 11-14.

Knafou, Rémi (2007), «L'urbain et le tourisme. Une construction laborieuse ", dans Philippe Duhamel et Rémi Knafou (dir.), Les mondes urbains du tourisme, Paris, Belin, p. 9-21.

Knafou, Rémy et Mathis Stock (2003), «Tourisme », dans Jacques Lévy et Michel Lussault (dir.), Dictionnaire de la géographie et de l'espace des sociétés, Paris, Belin, p. 931-933.

Knafou, Rémy, Mireille Bruston, Florence Deprest, Philippe Duhamel, Jean-Christophe Gay et Isabelle Sacareau (1997), "Une approche géographique du tourisme ", L'Espace géographique, tome 26, n 3, p. 193-204.

Kuhn, Thomas Samuel (1983), La structure des révolutions scientifiques, Paris, Flammarion.

Langevin, Érik (2000), «Le tourisme de l'An 2000... Avant Jésus-Christ », Téoros, vol. 19, nº 1 , p. 9-13.

Laplante, Marc (1999), «L'expérience touristique dans une société nomade ", Téoros, vol. 18, $n^{\circ} 3$, p. 17-21.
Laurent, Alain (1973), Libérer les vacances, Paris, Éditions du Seuil.

Leiper, Neil (2000), «An Emerging Discipline », Annals of Tourism Research, vol. 27, n 3, p. 805-809.

Le Moigne, Jean-Louis (1990), «Épistémologies constructivistes et sciences de l'organisation ", dans Alain Charles Martinet (dir.), Épistémologies et sciences de gestion, Paris, Economica, p. 81-140.

Le Moigne, Jean-Louis (2001), Le constructivisme, tome 3, Paris, L'Harmattan.

MacCannel, Dean (1976), The Tourist. A New Theory of the Leisure Class, New York, Schocken Books.

Martinet, Alain Charles (1990), " Grandes questions épistémologiques et sciences de gestion ", dans Alain Charles Martinet (dir.), Épistémologies et sciences de gestion, Paris, Economica, p. 9-29.

McKercher, Bob (1999), "A Chaos Approach to Tourism ", Tourism Management, vol. 20, p. 425-434.

Messadié, Gérard (2003), Le tourisme va mal. Achevons-le!, Paris, Éditions Max Milo.

Nowotny, Helga, Peter Scott et Michael Gibbons, (2003), Repenser la science: savoir et société à l'ère de l'incertitude, Paris, Belin, 2003.

Paquot, Thierry (2001), «Tourisme contre voyage. La tyrannie douce de l'air conditionné ", Le Monde diplomatique, [http://www.monde-diploma tique.fr/2001/07/PAQUOT/15344], consulté en janvier 2008.

Piaget, Jean (dir.) (1967), Logique et connaissance scientifique, Paris, Gallimard, 1967.

Pronovost, Gilles (1999), « Héritages et défis de la sociologie du loisir ", Revue Loisir et Société, vol. 22, no 1, p. 11-13.

Sacareau, Isabelle et Mathis Stock (2003), « Qu'est ce que le tourisme? ", dans Mathis Stock (dir.), Le tourisme - Acteurs, lieux et enjeux, Paris, Belin, p. 7-32.

Stafford, Jean (1985), « Le paradigme culturaliste en téorologie : étude, analyse et critique ", Téoros, vol. $7, n^{\circ} 1$, printemps, p. 5-8.

Stafford, Jean (1992), "Connaissances en tourisme et reconnaissance sociale ", Téoros, vol. 11, $n^{\circ} 1$, p. 44-46.

Stafford, Jean (2003), « La crise de la recherche en tourisme : la solution est dans les problèmes ", Revue Loisir et Société, vol. 26, n 1, p. 87-95.

Tribe, John (1997), «The Indiscipline of Tourism», Annals of Tourism Research, vol. 24, n०3, p. 638-657.

Tribe, John (2000), « Indisciplined and Unsubstantiated ", Annals of Tourism Research, vol. 27, $n^{\circ}$ 3, p. 809-813.

Urbain, Jean-Didier (1991), L'idiot du voyage, Paris, Éditions Plon.

Wieviorka, Michel (2007), "Introduction », dans Michel Wieviorka (dir.), Les sciences sociales en mutation, Paris, Éditions sciences humaines, p. 9-21. 